\title{
SPRI: A SIMULATOR OF POLARIMETRIC RADAR IMAGES
}

\author{
Roger D. De Roo \\ Fawwaz T. Ulaby \\ Gregory S. Samples \\ The University of Michigan, Radiation Lab \\ Ann Arbor, MI 48109-2122 USA \\ FAX: +1-734-647-2106 \\ email: deroo@umich.edu
}

\author{
John C. Costanza \\ Army Research Laboratory \\ Adelphi, MD 20783 USA
}

\begin{abstract}
A millimeter-wave $(M M W)$ radar clutter image synthesizer that will create faithful polarimetric clutter images is being developed as a suite of PCI software applications known as SPRI, the Simulator of Polarimetric Radar Images. SPRI will enable the user to create new clutter images from existing images, such as aerial photography or radar images from a sensor at a different frequency than that desired. The user can also create synthesized images from scratch. Since the techniques to implant hard targets are well developed, SPRI is expected to have application in automatic target recognition (ATR) development, sensor evaluation, and possibly in soldier training.

SPRI randomly generates a complex scattering matrix for each pixel in an image region which statistically satisfies a measured or derived Mueller matrix describing the clutter in that region. From the scattering matrix, radar cross sections per unit area of every pixel can be obtained for any transmit-receive polarization pair, as well as any other polarimetric quantity. The results of a fidelity test will be shown, where 35-GHz synthesized clutter is compared to the actual Lincoln Lab 35-GHz synthetic aperture radar (SAR) scene on which it is based. Examples are presented of a simulated clutter image at 35-GHz created from aerial photography, and a $95-\mathrm{GHz}$ clutter image created from $35-\mathrm{GHz}$ imagery.
\end{abstract}

\section{INTRODUCTION}

Radar images have proven to be invaluable tools in the re-

\footnotetext{
*Prepared through collaborative participation in the Advanced Sensors Consortium sponsored by the U.S. Army Research Laboratory under Cooperative Agreement DAAL01-96-2-0001. The U.S. Government is authorized to reproduce and distribute reprints for Government purposes notwithstanding any copyright notation thereon.
}

mote sensing community, with regard to both civilian and military applications. With the advent of Synthetic Aperture Radar (SAR), very high resolution images can be readily generated at both centimeter and millimeter wavelengths. For many tasks, such as testing Automatic Target Recognition (ATR) algorithms, it is desirable to have a particular radar image taken with a particular sensor under particular conditions. Such radar images are often unavailable, due to the fact that a radar observation like the one desired has never been performed, or because an existing image cannot be released. To make the observation would be very expensive, and even more so if the sensor has yet to be built.

Not all applications require real images. Training of personnel to read radar images, for example, do not require real images. The aforementioned ATR testing is another example where real images are not required. Evaluation of proposed sensors prior to prototyping is another application of synthesized imagery.

The majority of any radar image consists of clutter. Clutter behaves according to a particular statistical distribution, and this behavior is used to model the clutter. Remaining objects in an image consist of man-made targets which may or may not behave like clutter does. In SPRI, the Simulator of Polarimetric Radar Images, polarimetric clutter simulation will be combined with existing target injection techniques.

The clutter generation in SPRI depends on two parts: a clutter database and a simulation algorithm. The simulation algorithm converts random numbers into complex scattering matrices, $\mathbf{S}$, which are statistically appropriate for the clutter being modeled. The scattering matrix $\mathbf{S}$ is a $2 \times 2$ matrix given by

$$
\mathbf{S}=\left[\begin{array}{ll}
S_{v v} & S_{v h} \\
S_{h v} & S_{h h}
\end{array}\right]
$$

which relates the magnitude and phase of the scattered elec- 


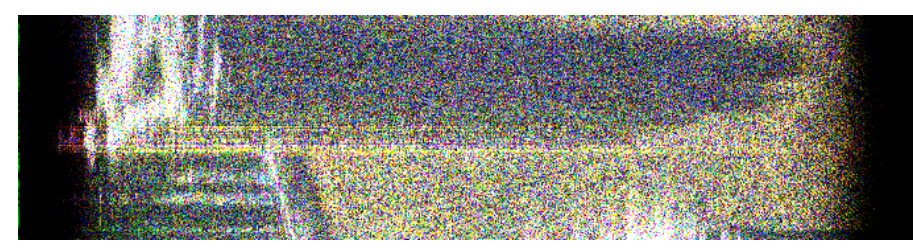

Figure 1: Single look Ka-band SAR image used for verifying the homogeneous simulation algorithm of SPRI. HH is in red, $\mathrm{VV}$ is in green and $\mathrm{VH}$ is in blue. The depression angle is $3.8^{\circ}$ and illumination is from the left. Trees in the upper left of the image are casting long shadows across the image.

tric field of either $v$ or $h$ polarization to that of the incident field at $v$ or $h$ polarization. But the conversion requires information on the covariance of the $\mathrm{S}$ matrix elements that is unique to the particular clutter being modeled. A clutter database of measured Mueller matrices for various types of clutter under different conditions provides the information required for the simulator algorithm.

\section{EXAMPLES OF SIMULATED IMAGES}

The imagery used was obtained by the Lincoln Lab $33.6 \mathrm{GHz}$ SAR [1] on behalf of the Army Research Laboratory. The image is one frame of many acquired near Hillsboro, MD, on the Eastern Shore, about $50 \mathrm{~km}$ East of Annapolis, MD, in the Spring of 1992. The particular image considered here is from mission 335, pass 5, level 4, frame 216. It has 2048 pixels in the range direction and 512 pixels in the azimuth direction; pixel resolution is $22.87 \mathrm{~cm}$ in both directions. The data are single look complex and fully polarimetric. This image is shown in Figure 1, and an aerial photograph of the same area taken at about the same time is shown in Figure 2. The PCI program DILL was used to read the 8-8-4 data and save the image to a PCI image database.

\section{A Fidelity Test: Simulation as a Lossy Compression}

The first image simulated will demonstrate two features of the most basic parts of SPRI: first, it will show how the algorithm is implemented in PCI, and second, it will show how faithful the simulation algorithm is in re-creating real data.

The first step is to segment the image into regions of reasonable homogeneity. This was achieved by converting the

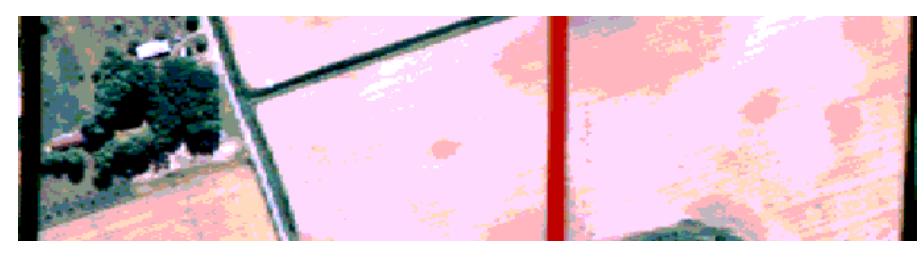

Figure 2: Aerial photo of region in the previous figure. This photograph and the image in the previous Figure are not coregistered. The vertical bands across the photo are artifacts.

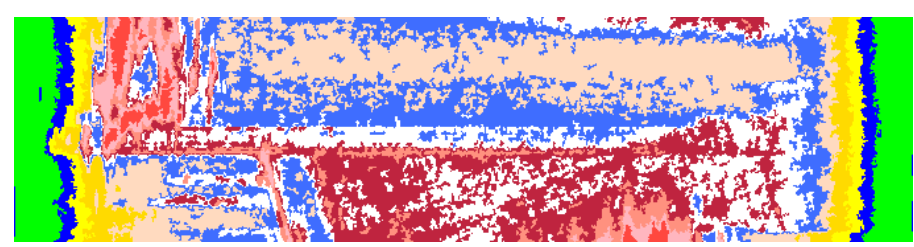

Figure 3: Regions extracted from the original radar image.

$\mathrm{VV}, \mathrm{HH}$, and $\mathrm{VH}$ amplitude channels to decibels, so that the fading variation would be constant across the image [2](PCI program LLOG), and successively applying an enhanced Lee adaptive despeckling filter [3](PCI program FELEE) on each of these channels. The resulting despeckled channels were used as the input to an unsupervised clustering algorithm [4](PCI program ISOCLUS), the output of which was sieved (PCI program SIEVE) to blend regions smaller than 32 pixels into their most appropriate neighbors. This process found 11 regions in the image, of which 4 are used in characterizing the intensity roll-off at each edge of the image in the range direction. For each region, the average Mueller matrix was calculated from the original single look data (PCI program MMMSLC). These regions are shown in Figure 3.

For a test of the simulation, these regions and the Mueller matrices extracted from them were used to reconstruct the radar image using the algorithm of Lee et al. [5] (PCI program SIMSLC). Three complex numbers of white Gaussian noise are generated for each pixel, as represented in Figure 4. These Gaussian noise numbers are multiplied by the appropriate eigenvalues and eigenvectors of the covariance matrices for the regions shown in Figure 3 to create the simulated image. The covariance matrix is a rearrangement of the information in the Mueller matrix. The resulting simulation is shown in Figure 5.

The match between the simulated image and the original im- 


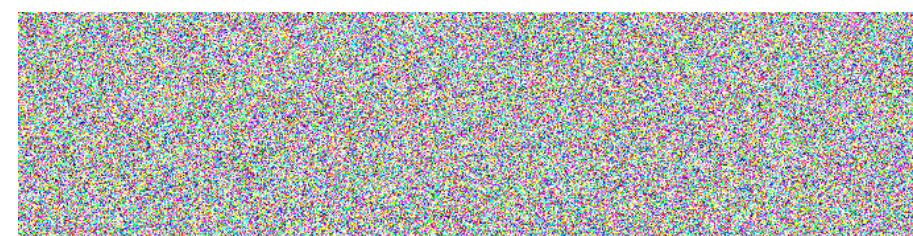

Figure 4: Three channels of noise used to simulate an image.

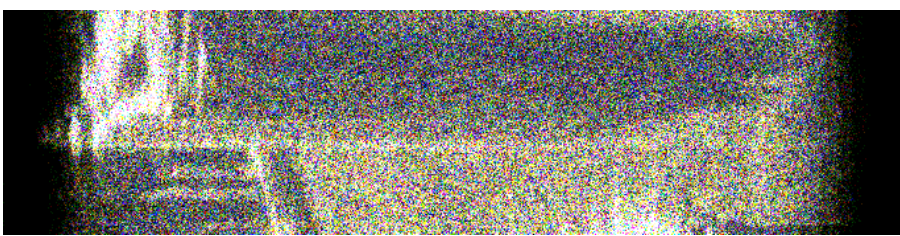

Figure 5: The simulated image generated from the noise in Figure 4 and the average Mueller matrices associated with each region in Figure 3.

age is very good overall, but the simulation is not an exact re-creation of the image in Figure 1. On a pixel by pixel basis, the original image and the simulated image are very different. But on a statistical basis, the two agree very closely, even for polarimetric quantities like co-polarized phase differences. One other way in which the original image and the simulated image differ is in the sensor artifacts: in the original image several streaks exist in range, due to a very large scatterers at the same azimuth position but just off to the left of the image. These streaks apparently do not obey Rayleigh statistics and thus are not simulated faithfully. Sensor degradation post-processing is required to properly create these image artifacts.

In a sense, this exercise results in a lossy compression of the full polarimetric image down to a simple bitmap and 110 real numbers (11 Mueller matrices with 10 independent numbers each). While the original and the simulated image match closely in their amplitude distributions, there is no guarantee that either image has the correct image intensity. That is, if there were any calibration errors in the original image given by Figure 1, the errors have been simulated in Figure 5 as well.

\section{Simulation from Aerial Photography}

As an additional example of the simulation algorithm, the aerial photograph of Figure 2 was used as the basis for gen-

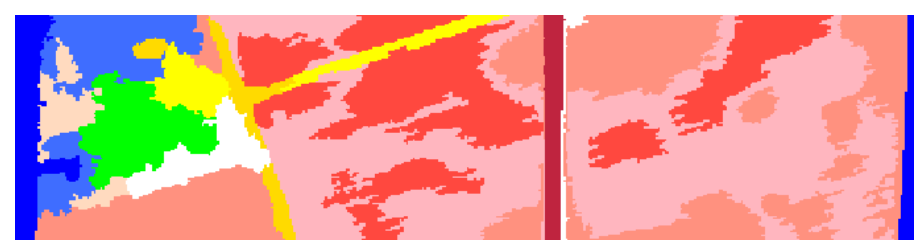

Figure 6: Regions extracted from the aerial photograph.

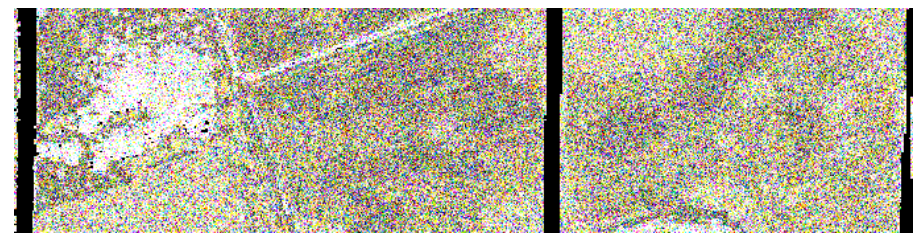

Figure 7: Simulated Ka-band image using the aerial photograph to determine the regions and the actual radar image to determine the Mueller matrices. Because the aerial photo was from directly overhead, the layout of the fields are correct, but the trees do not cast long shadows.

erating the regions to be filled with clutter. The segmentation of the photograph into regions is shown in Figure 6. Using these regions, and the same averaged Mueller matrices as used in the previous simulation, the image in Figure 7 is simulated. A striking difference between the actual image of Figure 1 and this simulation is observed: the trees do not cast shadows in this simulation. This is because the aerial photograph was from overhead, while the original image was taken from near grazing. Shadows must be added in this case; shadows will be incorporated in future versions of SPRI.

\section{Simulation from Ka-band to W-band}

A third example of a simulated image addresses the problem of translating a SAR scene observed at one frequency to a SAR scene at a different frequency. For this simulation, the regions are determined from the original SAR image as depicted in Figure 3, but the Mueller matrices were extracted from the University of Michigan millimeterwave phenomenology program [6], which includes data from the U.S. Department of Defense's Smart Weapons Operability Enhancement program [7]. For the shadow regions, the Mueller matrices for the trees were simply scaled down by a factor ranging between 2 and 10 . The resulting simulation appears in Figure 8. 


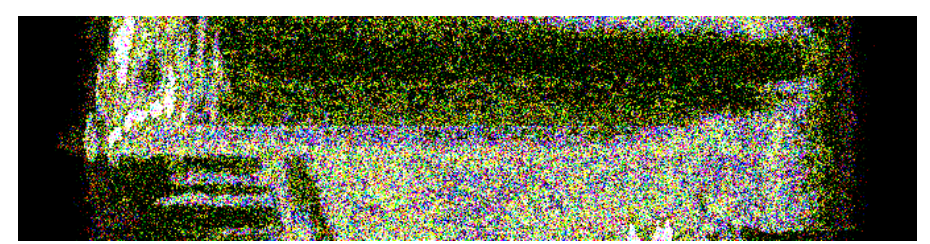

Figure 8: Simulated W-band image using the actual Kaband radar image to determine the regions and Mueller matrices from the University of Michigan's phenomenology program.

\section{CONCLUSIONS}

The University of Michigan has made the first component of SPRI: the Simulator of Polarimetric Radar Images. This first component of SPRI is a radar simulator which can create high-resolution polarimetric single-look clutter images. The simulator draws on a user-specified geometry and a database of Mueller matrices for the regions within that geometry to create radar clutter images. The user-specified geometry may be from an existing SAR image at a different radar frequency from that desired, an existing aerial photograph, or the user's imagination. The Mueller matrix database is being developed by the University of Michigan at $35 \mathrm{GHz}$ and $95 \mathrm{GHz}$, but existing data (ARL, Lincoln Lab) may also be used.

Future enhancements to SPRI includes the incorporation of shadows, texture, hard target implantation, and sensor degradation. A number of internal verifications are ongoing, to assure that the simulator produces clutter with the appropriate statistics. External tests can fall under two categories: an absolute test, in which a simulated image is checked against a real image, and a functional test, in which a simulated image is compared to a real image for its intended use as an Automatic Target Recognition algorithm testbed. In the absolute test, sufficient ground truth must be collected in conjunction with the acquisition of a real image. Such a test would be difficult to perform, as few real images have sufficient ground truth data associated with them. The functional test could be performed in the absence of sufficient ground truth by evaluating ATR approaches with known probability of detection and known false alarm rates on the simulation. The MIMEX dataset provides a unique opportunity to simulate millimeterwave images from aerial photography and from centimeterwave images, and to cross check these simulations against real millimeterwave images of the same scene.

\section{REFERENCES}

[1] J. C. Henry, T. J. Murphy, and K. M. Carusone, "The Lincoln Laboratory millimeter-wave synthetic aperture radar SAR imaging system," Proceedings of SPIE, vol. 1630, pp. 35-52, 1992.

[2] F. T. Ulaby, R. K. Moore, and A. K. Fung, Microwave Remote Sensing: Active and Passive, volume 3, pp. 1818-1819, Artech House, Norwood, MA, 1986.

[3] A. Lopes, R. Touzi, and E. Nezry, "Adaptive speckle filters and scene heterogeneity," IEEE Transactions on Geoscience and Remote Sensing, vol. 28, no. 6, pp. 992-1000, November 1990.

[4] J. T. Tou and R. C. Gonzalez, Pattern Recognition Principles, Addison-Wesley, Reading, MA, 1974.

[5] J. S. Lee, M. R. Grunes, and R. Kwok, "Classification of multi-look polarimetric SAR imagery based on complex Wishart distribution," International Journal of Remote Sensing, vol. 15, no. 11, pp. 2299-2311, 20 July 1994.

[6] F. T. Ulaby, A. Nashashibi, A. El-Rouby, E. S. Li, R. D. De Roo, K. Sarabandi, R. J. Wellman, and H. B. Wallace, "95-GHz scattering by terrain at near-grazing incidence," IEEE Transactions on Antennas and Propagation, vol. 46, no. 1, pp. 3-13, January 1998.

[7] J. P. Welsh, "Smart weapons operability enhancement (SWOE) joint test and evaluation (JT\&E) program final report," Technical Report SWOE Report 94-10, US Department of Defense, August 1994.

${ }^{*}$ The views and conclusions contained in this document are those of the authors and should not be interpreted as representing the official policies, either expressed or implied of the Army Research Laboratory or the U.S. Government. 